\title{
EXIT TIMES FOR ELLIPTIC DIFFUSIONS AND BMO
}

\author{
by R. BAÑUELOS AND B. ØKSENDAL
}

(Received 11th October, 1985)

In 1948 P. Lévy formulated the following theorem: If $U$ is an open subset of the complex plane and $f: U \rightarrow \mathbb{C}$ is a nonconstant analytic function, then $f$ maps a 2dimensional Brownian motion $B_{t}$ (up to the exit time from $U$ ) into a time changed 2-dimensional Brownian motion. A rigorous proof of this result first appeared in McKean [22]. This theorem has been used by many authors to solve problems about analytic functions by reducing them to problems about Brownian motion where the arguments are often more transparent. The survey paper [8] is a good reference for some of these applications. Lévy's theorem has been generalized, first by Bernard, Campbell, and Davie [5], and subsequently by Csink and Øksendal [7]. In Section 1 of this note we use these generalizations of Lévy's theorem to extend some results about BMO functions in the unit disc to harmonic morphisms in $\mathbb{R}^{n}$, to holomorphic functions in $\mathbb{C}^{n}$, and to analytic functions on Riemann surfaces. In Section 2, we characterize the domains in $\mathbb{R}^{n}$ which have the property that the expected exit time of elliptic diffusions is uniformly bounded as a function of the starting point. This extends a result of Hayman and Pommerenke [15], and Stegenga [24] about BMO domains in the complex plane.

\section{Exit times, area, and BMO}

First we recall the generalizations of Lévy's theorem that we will need.

1.1 (Bernard-Campbell-Davie [5]). Let $U \subset \mathbb{R}^{n}$ be an open set and $\phi=\left(\phi_{1}, \ldots, \phi_{m}\right)$ : $U \rightarrow \mathbb{R}^{m}$ be a $C^{2}$ function. Let $\left(B_{t}, \Omega, P_{x}\right)$ and $\left(\hat{B}_{t}, \widehat{\Omega}, \hat{P}_{x}\right)$ be Brownian motions in $\mathbb{R}^{n}$ and $\mathbb{R}^{m}$ respectively. Then (i) and (ii) below are equivalent:

(i) $\phi\left(B_{t}\right)$ is, up to the exit time $\tau=\tau_{U}$ from $U$, again Brownian motion in $\mathbb{R}^{m}$, except for a change in time scale. More precisely, if we define

$$
\sigma_{t}=\int_{0}^{t}\left|\nabla \phi_{1}\right|^{2} d s, \quad t \leqq \tau,
$$

then $\sigma_{t}$ is strictly increasing for a.a. $\omega, \phi^{*}(\omega)=\lim _{t \rightarrow \tau} \phi\left(B_{t}\right)$ exists a.e. on $\left\{\sigma_{\mathrm{t}}<\infty\right\}$, and the process $M_{t}(\omega, \hat{\omega}),(\omega, \hat{\omega}) \in \Omega \times \hat{\Omega}$, defined by

$$
M_{t}(\omega, \hat{\omega})= \begin{cases}\phi\left(B_{\sigma_{t}-1}\right) ; & t<\sigma_{\mathfrak{z}} \\ \phi^{*}(\omega)+\hat{B}_{t-\sigma_{\tau}} ; & t \geqq \sigma_{\tau}\end{cases}
$$

with probability law $P_{x} \times P_{0}$ coincides in law with Brownian motion in $\mathbb{R}^{m}$ starting at $\phi(x)$. 
(ii) The coordinate functions $\phi_{i}$ are all harmonic and their gradients $\nabla \phi_{i}$ are orthogonal to each other and have the same length.

It is not hard to show that the class of function satisfying (ii) agrees with the class of functions satisfying:

(iii) $h$ harmonic on $W$ (open) $\subset \mathbb{R}^{m} \Rightarrow h \circ \phi$ harmonic on $\phi^{-1}(W)$.

The functions satisfying (iii) are called harmonic morphisms and they have been studied by many authors in more general settings. See [11] and references there.

Now suppose $f: U \subset \mathbb{C}^{n} \rightarrow \mathbb{C}$ is a holomorphic function. Then the Cauchy-Riemann equations show that $f$ satisfies (ii) and therefore $f$ maps Brownian motion in $\mathbb{C}^{n}$ to Brownian motion in $\mathbb{C}$. When $n=1$, (ii) characterizes the analytic and conjugate analytic functions.

A further generalization of Lévy's theorem was given in [7] by Csink and Øksendal. We will briefly recall a special case of this result and refer the reader to their paper for details and generalizations. Let $A$ be a second order partial differential operator in $\mathbb{R}^{m}$ of the form

$$
A=\sum_{i j} a_{i j}(x) \frac{\partial^{2}}{\partial x_{i} \partial x_{j}}+\sum_{i} b_{i}(x) \frac{\partial}{\partial x_{i}}
$$

where we assume (1) $a_{i j}, b_{i} \in C^{\infty}$, (2) $a_{i j}=a_{j i}$, and (3) for all nonzero vectors $y \in \mathbb{R}^{m}$, $\sum_{i j} y_{i} a_{i j}(x) y_{j}>0$. By an Itô diffusion $X_{i}$ starting at $x$ with generator $A$ we shall mean a solution of the stochastic differential equation

$$
d X_{t}=\sigma\left(X_{t}\right) d B_{t}+b\left(X_{t}\right) d t, X_{0}=x
$$

Here $b=\left(b_{i}\right)$ and $\sigma$ is the matrix satisfying $\frac{1}{2} \sigma \sigma^{T}=a=\left(a_{i j}\right)$, where $\sigma^{T}$ is the transpose of $\sigma$. For the construction of the process see [16]. We have:

1.4 (Csink-Øksendal [7]). Let $X_{t}$ and $\hat{X}_{t}$ be two Itô diffusions on open subsets $U \subset \mathbb{R}^{n}, V \subset \mathbb{R}^{m}$ respectively. Let $A$ and $\hat{A}$ be the corresponding generators. Suppose $\phi: U \rightarrow V$ is $C^{2}$ and not $X_{t}$-finely locally constant. Then the following are equivalent:

(i) $\phi$ maps $X_{t}$ into a time change of $\hat{X}_{t}$.

(ii) There exists a continuous function $\lambda(x) \geqq 0$ on $U$, with $\lambda(x)>0$ except possibly on an $X_{t}$-finely nowhere dense set, such that for all $x \in U$ and $f \in C^{2}(V)$

$$
A[f \circ \phi](x)=\lambda(x) A[f](\phi(x))
$$

The function $\lambda$ gives the rate of time change to be used. In other words, if

$$
\sigma_{t}=\int_{0}^{t} \lambda\left(X_{s}\right) d s, \quad \text { for } \quad t<\tau=\tau_{U}
$$

then the process defined by 


$$
M_{t}(\omega, \hat{\omega})= \begin{cases}\phi\left(X_{\sigma_{\mathfrak{t}}-1}\right) ; & t<\sigma_{\mathfrak{\tau}} \\ \hat{X}_{\mathrm{t}-\sigma_{\mathfrak{\tau}}} ; & t \geqq \sigma_{\mathfrak{\tau}}\end{cases}
$$

with a natural probability law agrees in law with $X_{t}$. In fact, in this setting (ii) $\Leftrightarrow$ (i) without the condition that $\lambda>0$ except possibly on an $X_{t}$-finely nowhere dense set. See Theorem 3 in [23].

Next recall that a function $f: T=\{z:|z|=1\} \rightarrow \mathbb{C}$ is in BMO (bounded mean oscillation) if there is a constant $c$ such that $1 /|I| \int_{I}\left|f(\theta)-f_{I}\right|^{2} d \theta \leqq c^{2}$ where $f_{I}=1 /|I| \int_{I} f(\theta) d \theta$. The BMO norm of $f,\|f\|_{\text {BMO }}$, is the smallest constant $c$ for which this holds. By BMOA we shall mean the analytic functions in the unit disc $D$ with boundary values in BMO. Using basic properties about the Poisson kernel it can be shown that $f \in \mathrm{BMOA}$ if and only if

$$
\sup _{z \in D} \int_{T}|f(\theta)-f(z)|^{2} d \mu_{z}(\theta)<\infty
$$

where $\mu_{z}$ is the harmonic measure at $z$. (For the proof of (1.6) see [13]). If we translate (1.6) to probability language we see that $f \in \mathrm{BMOA}$ if and only if

$$
\sup _{z \in D} E_{z}\left|f\left(B_{\tau}\right)-f(z)\right|^{2}<\infty
$$

This probabilistic characterization of BMOA can be extended to define more generally a BMO norm on harmonic morphisms $\phi: U \rightarrow \mathbb{R}^{m}$, for an arbitrary open set $U \subset \mathbb{R}^{n}:$

Definition 1.1. Let $\phi: U \subset \mathbb{R}^{n} \rightarrow \mathbb{R}^{m}$ be a $C^{2}$ harmonic morphism. Then we say that $\phi \in \mathrm{BMO}(\mathrm{U})$ if $\sigma_{\mathrm{\tau}}=\int_{0}^{\tau}\left|\nabla \phi_{1}\right|^{2} d s<\infty$ a.e. $P_{x}$ for all $x \in U$ and

$$
\|\phi\|_{\mathrm{BMO}(U)}^{2}=\sup _{x \in U} E_{x}\left|\phi^{*}(\omega)-\phi(x)\right|^{2}<\infty .
$$

Here $\phi^{*}(\omega)=\lim _{t \rightarrow \tau} \phi\left(B_{t}\right)$ is the stochastic boundary function of $\phi$ whose existence is given by the Bernard, Campbell, and Davie extension of Lévy's theorem. Note that (1.8) agrees with (1.7) in the case of analytic functions in the unit disc.

Theorem 1.2. Let $\phi: U \subset \mathbb{R}^{n} \rightarrow \mathbb{R}^{m}$ be a harmonic morphism. Then

$$
\|\phi\|_{\mathrm{BMO}(U)}^{2} \leqq m \cdot \sup _{y \in \phi(U)} E_{y}\left(\tau_{\phi(U)}\right)
$$

where $\tau_{\phi(U)}$ is the first exit time from $\phi(U)$.

Proof. Using the Itô formula we have

$$
\begin{aligned}
\|\phi\|_{\mathrm{BMO}(U)}^{2} & =\sup _{x \in U} E_{x}\left[\left|\phi^{*}(\omega)-\phi(x)\right|^{2}\right] \\
& =\sum_{i=1}^{m}\left(\sup _{x \in U} E_{x}\left[\left|\int_{0}^{\tau} \nabla \phi_{i}\left(B_{s}\right) \cdot d B_{s}\right|^{2}\right]\right)
\end{aligned}
$$




$$
\begin{aligned}
& =\sum_{i=1}^{m}\left(\sup _{x \in U} E_{x}\left(\int_{0}^{\tau}\left|\nabla \phi_{i}\left(B_{s}\right)\right|^{2} d s\right)\right) \\
& =m \sup _{x \in U} E_{x}\left(\int_{0}^{\tau}\left|\nabla \phi_{1}\left(B_{s}\right)\right|^{2} d s\right) \\
& =m \sup _{x \in U} E_{x}\left[\sigma_{t}\right] \leqq m \sup _{y \in \phi(U)} E_{y}\left[\tau_{\phi(U)}\right]
\end{aligned}
$$

since $\sigma_{\tau} \leqq \tau_{\phi(U)}$ by the Bernard-Campbell-Davie theorem.

If $f$ is an analytic function in the unit disc $D$ such that the area of $f(D)$ (counting multiplicities) is finite, then it is easy to show that $f \in \mathrm{BMOA}$. If one ignores multiplicities the situation is more difficult. It was proved by Alexander, Taylor, and Ullman [2] that if area $(f(D))<\infty$, then $f \in H^{2}$. This was improved by Hansen [14] who showed that area $(f(D))<\infty$ implies $f \in H^{p}$ for all $p<\infty$. Finally, Stegenga [24] showed that if area $(f(D))<\infty$, then $f \in$ BMOA. In [4] Axler and Shapiro gave a new proof of Stegenga's result using Alexander's spectral area estimates and extended their result to the ball in $\mathbb{C}^{n}$. Our stochastic approach gives the following generalization of these results:

Theorem 1.3. Let $U \subset \mathbb{R}^{n}$ be open and let $\phi: U \rightarrow \mathbb{R}^{m}$ be a harmonic morphism. Then

$$
\|\phi\|_{\mathrm{BMO}(U)} \leqq\left(\frac{\operatorname{vol}(\phi(U))}{c_{m}}\right)^{1 / m}
$$

where

$$
c_{m}=\frac{2 \pi^{m / 2}}{m \Gamma(m / 2)}
$$

is the volume of the unit ball in $\mathbb{R}^{m}$.

Proof. Let $D(r)=\left\{x \in \mathbb{R}^{m}:|x|<r\right\}$ be the ball in $\mathbb{R}^{m}$ such that $\operatorname{vol}(D(r))=\operatorname{vol}(\phi(U))$. Let $B_{t}$ be a Brownian motion in $\mathbb{R}^{m}$ starting at 0 and let $\tau_{D(r)}=\inf \left\{t>0: B_{t} \notin D(r)\right\}$. It follows from Aizenman and Simon [1] that for any $y \in \phi(U)$,

$$
E_{y}\left(\tau_{\phi(U)}\right) \leqq E_{0}\left(i_{D(r)}\right) \text {. }
$$

Thus $\|\phi\|_{\mathrm{BMO}(U)}^{2} \leqq m E_{0}\left(\tau_{\mathrm{B}(r)}\right)$. Using the Itô formula on the function $f(y)=|y|^{2}, y \in \mathbb{R}^{m}$, we can show that

$$
E_{0}\left(\tau_{D(r)}\right)=\frac{r^{2}}{m}
$$




$$
\|\phi\|_{\mathrm{BMO}(U)}^{2} \leqq r^{2}=\left(\frac{\operatorname{vol}(\phi(U))}{c_{m}}\right)^{2 / m}
$$

and we have the theorem.

Remark 1.4. Note that the inequality (1.9) is sharp. This can be seen by choosing $U$ to be the cylinder $U=D(0,1) \times \mathbb{R}^{n-m}$, where $D(0,1)$ is the unit ball in $\mathbb{R}^{m}$, and $\phi\left(x_{1}, \ldots, x_{n}\right)=\left(x_{1}, \ldots, x_{m}\right)$.

Let $U$ be an open set in $\mathbb{R}^{m}$ which has a Green function $g_{U}(x, y)$. As is well known (see [10] or [16])

$$
E_{x}\left(\tau_{v}\right)=\int_{U} g_{U}(x, y) d y, \quad \forall x \in U
$$

If we assume $U$ has finite volume then the estimates above show that

$$
E_{x}\left(\tau_{U}\right) \leqq \frac{1}{m}\left(\frac{\operatorname{vol}(U)}{c_{m}}\right)^{2 / m}
$$

and we have:

Corollary 1.5. Suppose $U$ is an open set in $\mathbb{R}^{m}$ with finite volume and with Green function $g_{U}(x, y)$. Then

$$
\int_{U} g_{U}(x, y) d y \leqq \frac{1}{m}\left(\frac{\operatorname{vol}(U)}{c_{m}}\right)^{2 / m}, \quad \forall x \in U
$$

Note: By the Green function $g_{U}(x, y)$ we mean the fundamental solution of $\frac{1}{2} \Delta$ with zero boundary conditions.

With little effort we can extend Corollary 1.5 to Green functions for uniformly elliptic operators in divergence form. More precisely, suppose our operator $A$ in (1.2) has the form

$$
A=\sum_{i j=1}^{m} \frac{\partial}{\partial x_{i}}\left(a_{i j} \frac{\partial}{\partial x_{j}}\right)
$$

with the coefficients satisfying (1) and (2) of (1.2) and, in addition, there exists a constant $\lambda>1$ such that for all $(x, y) \in \mathbb{R}^{m} \times \mathbb{R}^{m}$

$$
\lambda^{-1}|y|^{2} \leqq \sum_{i j=1}^{m} a_{i j}(x) y_{i} y_{j} \leqq \lambda|y|^{2}
$$

Theorem 1.6. Suppose $U$ is an open set in $\mathbb{R}^{m}$ with finite volume. Let $g \hat{U}_{u}^{A}(x, y)$ be the Green function for $A$ in $U$. Then there exists a constant $K_{\lambda, m}$ depending only on the 
ellipticity constant $\lambda$ and the dimension $m$ such that

$$
\int_{U} g_{U}^{A}(x, y) d y \leqq K_{\lambda, m}(\operatorname{vol}(U))^{2 / m}
$$

for all $x \in U$.

Proof. Let $X_{t}$ be the Itô diffusion corresponding to $A$. Let $\tau=\inf \left\{t>0: X_{t} \notin U\right\}$. Then

$$
E_{x}(\tau)=\int_{U} g_{U}^{A}(x, y) d y
$$

Let $P_{t}(x, y)$ be the transition probabilities for $X_{t}$. As is well known $([16]), P_{t}(x, y)$ is the fundamental solution of the heat equation $d / d t-A$. By Aronson ([3]) there exist constants $K_{\lambda, m}$ and $R_{\lambda, m}$ such that

$$
P_{t}(x, y) \leqq K_{\lambda, m} \frac{1}{t^{m / 2}} e^{-R_{\lambda, m}} \frac{|x-y|^{2}}{t}
$$

for all $(t, x, y) \in(0, \infty) \times \mathbb{R}^{m} \times \mathbb{R}^{m}$. So

$$
P_{x}(\tau>t) \leqq P_{x}\left(X_{t} \in U\right)=\int_{U} P_{t}(x, y) d y \leqq K_{\lambda, m} \frac{1}{t^{m / 2}} \int_{U} e^{-R_{\lambda, m}} \frac{|x-y|^{2}}{t} d y \leqq K_{\lambda, m} \frac{1}{t^{m / 2}} \operatorname{vol}(U) .
$$

Therefore, if $m>2$,

$$
\begin{aligned}
E_{x}(\tau) & =\int_{0}^{\infty} P_{x}(\tau>t) d t \leqq \int_{0}^{\infty} \min \left\{1, K_{\lambda, m} \cdot \operatorname{vol}(U) \cdot t^{-m / 2}\right\} d t \\
& =\left[K_{\lambda, m} \cdot \operatorname{vol}(U)\right]^{2 / m}+\int_{\left(K_{\lambda, m} \cdot \operatorname{vol}(U)\right)^{2 / m}}^{\infty} K_{\lambda, m} \operatorname{vol}(U) t^{-m / 2} d t=K_{\lambda, m}(\operatorname{vol}(U))^{2 / m}
\end{aligned}
$$

If $m=2$ we refine the estimate (1.17) as follows (put $K_{\lambda, z}=K, R_{\lambda, z}=R$ ):

$$
\begin{aligned}
P_{x}(\tau>t) & \leqq \frac{K}{t} \int_{U} \exp \left(-R \cdot \frac{|x-y|}{t}\right)^{2} d y \leqq \frac{K}{t} \cdot\left\{y ;|x-y|<\sqrt{\frac{\operatorname{vol}(U)}{\pi}}\right\} \int \exp \left(-R \cdot \frac{|x-y|}{t}\right)^{2} d y \\
& =\frac{2 \pi K}{t} \cdot \int_{0}^{\sqrt{\operatorname{vol}(U) / \pi}} \exp \left(-R \cdot \frac{r^{2}}{t}\right) r d r \\
& =\frac{\pi K}{R} \cdot\left[1-\exp \left(-R \cdot \frac{\operatorname{vol}(U)}{\pi t}\right)\right] .
\end{aligned}
$$

Then by the strong Markov property we have 


$$
P_{x}(\tau>2 t) \leqq\left(\sup _{x \in U} P_{x}(\tau>t)\right)^{2} \leqq \frac{\pi^{2} K^{2}}{R^{2}}\left(1-\exp \left(-\frac{R \cdot \operatorname{vol}(U)}{\pi t}\right)\right)^{2}
$$

so that

$$
P_{x}(\tau>s \cdot \operatorname{vol}(U)) \leqq \frac{\pi^{2} K^{2}}{R^{2}}\left(1-\exp \left(-\frac{2 R}{\pi s}\right)\right)^{2}
$$

Hence

$$
\begin{aligned}
\int_{0}^{\infty} P_{x}(\tau>t) d t & =\int_{0}^{\infty} P_{x}(\tau>s \cdot \operatorname{vol}(U)) \operatorname{vol}(U) \cdot d s \\
& \leqq \frac{\pi^{2} K^{2} \cdot \operatorname{vol}(U)}{R^{2}} \cdot \int_{0}^{\infty}\left(1-\exp \left(-\frac{2 R}{\pi s}\right)\right)^{2} d s=\frac{2 \pi K^{2} \cdot \operatorname{vol}(U)}{R} \int_{0}^{\infty}\left(1-e^{-u}\right)^{2} u^{-2} d u \\
& =K_{\lambda, 2} \cdot \operatorname{vol}(U),
\end{aligned}
$$

which completes the proof.

Next, we consider the standard complex $n$-dimensional Euclidean space $\mathbb{C}^{n}$ and operators of the form

$$
A=4 \sum_{i j} a_{i j}(z) \frac{\partial^{2}}{\partial z_{i} \partial \bar{z}_{j}}
$$

where the coefficients $a_{i j}$ satisfy (1), (2), and (3) of (1.2). The diffusion associated to $A$ will be denoted by $B_{i}^{A}$. In the case when $a_{i j}=\delta_{i j},\left(\delta_{i j}=1\right.$ if $i=j$ and 0 otherwise), we get the ordinary Euclidean Brownian motion in $\mathbb{R}^{2 n}$ which we simply denote by $B_{t}$.

Lemma 1.7. Let $U$ be an open set in $\mathbb{C}^{n}$ and let $\phi: U \rightarrow \mathbb{C}$ be a holomorphic function. Then for all $f \in C^{2}(\mathbb{C})$ we have

$$
A[f \circ \phi]=\left(\sum_{i j} a_{i j} \frac{\partial \phi}{\partial z_{i}} \overline{\frac{\partial \phi}{\partial z_{j}}}\right) \cdot \frac{1}{2} \Delta(f) \circ \phi
$$

where $\Delta=4 \partial^{2} / \partial z \partial \bar{z}$ is the usual Euclidean Laplacian in $\mathbb{C}$. Therefore $\phi$ maps $B_{t}^{A}$ into a time change of Brownian motion $B_{1}$ in $\mathbb{C}$, with time change rate

$$
\lambda(z)=\sum_{i j} a_{i j}(z) \frac{\partial \phi}{\partial z_{i}} \frac{\overline{\partial \phi}}{\partial z_{j}} .
$$

Proof. The formula (1.19) can be easily verified by using the chain ruse. Ine conclusion of the lemma then follows from the Csink-Øksendal extension ((1.4) above) of Lévy's theorem. 
Assume $U$ is open in $\mathbb{C}^{n}$ and let $\tau_{U}^{A}$ be the first exit time of $B_{t}^{A}$ from $U$. If $\phi: U \rightarrow \mathbb{C}$ is holomorphic we will say that $\phi \in \operatorname{BMO}\left(U, B^{A}\right)$ if

$$
\sigma_{\tau A}=\int_{0}^{\tau_{U}^{A}} \lambda\left(B_{s}\right) d s<\infty \text { a.s. } P_{z} \text { for all } z \in U \text { and }\|\phi\|_{\mathrm{BMO}\left(U, B^{A}\right)}^{2}=\sup _{z \in U} E_{z}\left|\phi^{*}(\omega)-\phi(z)\right|^{2}<\infty,
$$

where $\phi^{*}(\omega)=\lim _{t \rightarrow \tau_{\hat{U}}} \phi\left(B_{t}^{A}\right)$, which exists on $\left\{\sigma_{\tau A}<\infty\right\}$, by Lemma 1.7. With these BMO-norms and the same argument used to prove Theorem 1.3 we have:

Theorem 1.8. Let $\phi: U \rightarrow \mathbb{C}$ be a holomorphic function and assume Area $(\phi(U))$ is finite. Then $\phi \in \mathrm{BMO}\left(U, B^{A}\right)$, for any $A$ and

$$
\|\phi\|_{\mathrm{BMO}\left(U, B^{A}\right)} \leqq\left(\frac{\operatorname{Area}(\phi(U))}{\pi}\right)^{1 / 2}
$$

If $U$ is a bounded strictly pseudoconvex domain in $\mathbb{C}^{n}$ with smooth boundary (see [25] for definitions) and we take as our operator $A$ the Euclidean Laplacian, then the BMO-space we obtain from (1.21) is the usual BMO-space associated with Euclidean balls. If we take the Laplacian of the Bergman metric as our operator $A$, we obtain the nonisotropic BMO-spaces associated with the skewed balls (see [18]). Thus Theorem 1.8 includes the extensions of Stegenga's result given by Axler and Shapiro [4] to the unit ball in $\mathbb{C}^{n}$.

Next we mention a result for Riemann surfaces which also follows from these methods. Let $R$ be a Riemann surface which possesses a Green function $g(x, a)$. Define by $\mathrm{BMO} A(R)$ the space of analytic functions on $R$ for which

$$
\|f\|_{\mathrm{BMOA}(R)}^{2}=\sup _{a \in R} \int_{R}\left|f^{\prime}(z)\right|^{2} g(a, z) d z<\infty .
$$

Since Lévy's theorem is also true for Riemann surfaces (see [17] and [22]) we have by the same arguments above:

Theorem 1.9. $\|f\|_{\mathrm{BMOA}(R)} \leqq\left(\frac{\text { Area } f(R)}{\pi}\right)^{1 / 2}$.

Note: This Theorem is the main result in [19].

Our final application of the above generalizations of Lévy's theorem is to asymptotic values of holomorphic function in weakly pseudoconvex domains. Let $U$ be a weakly pseudoconvex domain in $\mathbb{C}^{n}$ defined by $U=\left\{z \in \mathbb{C}^{n}: q(z)<0\right\}$ where $q \in C^{3}(\bar{U})$ is plurisubharmonic in $U$ and $\nabla q \neq 0$ on $\partial U$. Define $p(z)=\sum_{i=1}^{n}\left|z_{i}\right|^{2}-r^{2}$ where $r$ is chosen so large that $D(0, r) \supset \bar{U}$. Debiard and Gaveau [9] then consider the diffusion $B_{t}^{A}$ where the coefficients of $A$ are given by

$$
a_{i j}=-\frac{\partial^{2}}{\partial z_{i} \partial \bar{z}_{j}}(\log (p q))
$$


They show that $B_{t}^{A}$ has an infinite lifetime and that $B_{\infty}^{A}=\lim _{t \rightarrow \infty} B_{t}^{A} \in \partial U$ exists a.s. Moreover, the harmonic measure $\mu_{z_{0}}$ with respect to the process $B_{i}^{A}$ defined by $\mu_{z_{0}}(F)=$ $P_{z_{0}}\left(B_{\infty}^{A} \in F\right), F$ á Borel set in $\partial U$, has no mass on the set $W$ of weakly pseudoconvex boundary points of $U$. (see [9], Théorème 1). With these definitions we have

Theorem 1.10. Let $\phi: U \rightarrow \mathbb{C}$ be a holomorphic function. Suppose $\operatorname{Cap}(\mathbb{C} \backslash \phi(U))>0$ and that $\phi$ is proper, i.e. $K$ compact in $\phi(U) \Rightarrow \phi^{-1}(K)$ compact in $U$. Then almost all boundary points of $\phi(U)$ with respect to Euclidean harmonic measure for $\phi(U)$ are asymptotic values of $\phi$ at points in $\partial U \backslash W$.

Note: We say that $y$ is an asymptotic value of $\phi$ at $x \in \partial U$ if there exists a curve $\gamma:[0,1) \rightarrow U$ terminating at $x$ such that $y=\lim _{t \rightarrow 1} \phi\left(\gamma_{t}\right)$. By $\operatorname{Cap}(F)$ we mean the logarithmic capacity of the set $F$.

Proof. Let $\left(\bar{B}_{t}, \Omega, \bar{P}_{y}\right)$ be a Brownian motion in $\mathbb{C}$. The Euclidean harmonic measure $v_{y}$ for $\phi(U)$ at $y$ is supported by any set $H$ such that

$$
\bar{P}_{y}\left[\bar{B}_{\tau_{\phi(U)}} \in H\right]=1 .
$$

Let $\Omega_{0}=\left\{\omega: B_{\infty}^{A}(\omega)=\lim _{t \rightarrow \infty} B_{t}^{A}(\omega) \in \partial U \backslash W \& \phi^{*}(\omega)=\lim _{t \rightarrow \infty} \phi\left(B_{t}^{A}\right) \quad\right.$ exists $\} . \quad$ By the Debiard-Gaveau result and (1.4) we know that $P_{y}\left(\Omega_{0}\right)=1$ and that the process defined by

$$
M_{t}(\omega, \hat{\omega})= \begin{cases}\phi\left(B_{\left.\sigma_{t}-1\right)}^{A} ;\right. & t<\sigma_{\infty} \\ \phi^{*}+\hat{B}_{t} ; & t \geqq \sigma_{\infty}\end{cases}
$$

with probability law $P_{x} \times \hat{P}_{0}$ coincides with Brownian motion starting from $y=\phi(x)$. Since $\phi\left(B_{t}^{A}\right) \in \phi(U)$ for all $t<\infty$ and $\phi^{*} \in \partial(\phi(U))$ (since $\phi$ is proper) we conclude that $M_{\tau_{\phi(U)}}=\phi^{*}$ a.s. and therefore, if we put

$$
H=\left\{\phi^{*}(\omega): \omega \in \Omega_{0}\right\}
$$

we have $P_{x} \times \hat{P}_{0}\left\{M_{\mathrm{t}(U)} \in H\right\}=1$. Since all points of $H$ are of the form $\phi^{*}(\omega)=$ $\lim _{t \rightarrow \infty} \phi\left(B_{t}^{A}\right)$ where $B_{\infty}^{A}=\lim _{t \rightarrow \infty} B_{t}^{A} \in \partial U \backslash W$, the theorem is proved.

We end this section with some remarks. Let us assume that $U$ is a bounded strictly pseudoconvex domain in $\mathbb{C}^{n}$ with smooth boundary and let $\phi: U \rightarrow \mathbb{C}$ be a holomorphic function. Let us write $\|\phi\|_{\text {BMO }}$ for the BMO-norm in (1.21) with respect to either the Euclidean Laplacian or the Laplacian of the Bergman metric in $U$. It would be interesting to find necessary and sufficient conditions on $\phi$ in order to have equality in (1.22), i.e. so that

$$
\|\phi\|_{\text {вмO }}=\left(\frac{\operatorname{Area} \phi(U)}{\pi}\right)^{1 / 2} .
$$

Clearly if $U$ is the unit disc $D$ in the plane and $\phi$ is a Möbius transformation, we have equality. But we can have (1.26) with more complicated $\phi^{\prime}$ s. Let $\phi: U \rightarrow \mathbb{C}$ be an inner 
function. (The existence of inner functions in smoothly bounded pseudoconvex domains is shown in [21]). Since $\phi$ maps into the unit disc we have Area $\phi(U) \leqq \pi$. It is also clear that $\|\phi\|_{\mathrm{BMO}}^{2}=\sup _{z \in U}\left(1-|\phi(z)|^{2}\right)$. Thus to prove equality for inner functions we need to show $\sup _{z \in U}\left(1-|\phi(z)|^{2}\right)=1$. This follows from the following lemma which in the case of the unit disc in the complex plane is a special case of a theorem of Frostman, (see [13], p. 79).

Lemma 1.11. Let $U$ be a bounded strictly pseudoconvex domain in $\mathbb{C}^{n}$ with smooth boundary and let $\phi: U \rightarrow \mathbb{C}$ be an inner function. Then $\phi$ assumes every point in the unit disc except at most a set of capacity zero.

Proof. First we recall that from probabilistic potential theory (see [12]), if $K$ is a set of positive capacity in the unit disc $D$, then Brownian motion started at any point in $D$ will hit $K$ with positive probability before it exits $D$. If $B_{t}$ is a Brownian motion in $U$ run until it exists $U$, then we know that $\phi\left(B_{t}\right)$ is a time change of Brownian motion in the unit disc $D$. Since $\phi$ is an inner function, this Brownian motion is run for the same amount of time as the killed Brownian motion in $D$. So $\phi(U)$ cannot omit a set of positive capacity and the lemma is proved.

By carefully examining the proof of Theorem 1.3, Lemma 1.11, and the conditions for equality in the Aizenman-Simon result we used to prove Theorem 1.3, the interested reader can verify that if $U$ is an open set in $\mathbb{R}^{n}, D(1)$ is the unit ball in $\mathbb{R}^{m}$, and $\phi: U \rightarrow D(1)$ is a harmonic morphism, then equality holds in (1.9) if and only if $\phi$ is a stochastic inner function in the sense of Bernard-Campbell-Davie [5]. An example in [5] of a stochastic inner function which is not an inner function in the sense of complex analysis is the function $\phi$ mapping the unit ball in $\mathbb{R}^{4}$ to the unit ball in $\mathbb{R}^{3}$ given by $\phi\left(x_{1}, x_{2}, x_{3}, x_{4}\right)=\left(x_{1}^{2}+x_{2}^{2}-x_{3}^{2}-x_{4}^{2}, 2\left(x_{1} x_{3}-x_{2} x_{4}\right), 2\left(x_{1} x_{4}+x_{2} x_{3}\right)\right)$. The reader can easily verify that $\phi$ is a harmonic morphism and $|\phi(x)|=|x|^{2}$.

\section{Exit times, capacity, and BMO}

Hayman and Pommerenke [15] and independently Stegenga [24] discovered a capacity criterion for the domain $V \subset \mathbb{C}$ with the property that an analytic function $\phi$ defined on the unit disc $D$ with values in $V$ will belong to BMOA. More precisely, they showed that every such $\phi$ is in BMOA if and only if there exist constants $R$ and $\delta>0$ such that $\operatorname{Cap}\left\{D\left(x_{0}, R\right) \backslash V\right\}>\delta$ for all $x_{0} \in V$ where $D\left(x_{0}, R\right)=\left\{x \in \mathbb{C}:\left|x-x_{0}\right|<R\right\}$ and Cap denotes the logarithmic capacity. In this section we show that this capacity condition can be used to characterize the domains in $\mathbb{R}^{n}$ with the property that the expected exit time of uniformly elliptic diffusions is uniformly bounded as a function of the starting point. This, combined with Theorem 1.2 of Section 1, gives an extension of (one part of) the Hayman-Pommerenke-Stegenga result to harmonic morphisms in $\mathbb{R}^{n}$.

For the rest of the paper $A$ will be an operator satisfying the conditions in (1.13) and (1.14) and with associated diffusion $B_{t}^{A}$. If $V$ is an open set in $\mathbb{R}^{n}$ we denote by $g_{V}(x, y)$ the Green function of the operator $A$ for the set $V$. If $F$ is a subset of $V$ we define

$$
\operatorname{Cap}_{v}(F)=\sup \left\{\mu(F): \mu \text { is a Borel measure supported in } F \text { and } \int_{F} g_{v}(x, y) d \mu(y) \leqq 1 \forall x\right\} \text {. }
$$

The equilibrium measure of $F$ is denoted by $\mu_{F, V}$. 
Theorem 2.1. Let $V$ be an open set in $\mathbb{R}^{n}$ and let $\tau$ be the first exit time of $B_{i}^{A}$ from $V$. Then $\sup _{x \in V} E_{x}(\tau)<\infty$ if and only if there exists an $R$ and $a \delta>0$ such that $\operatorname{Cap}_{D(x, 2 R)}(D(x, R) \backslash V) \geqq \delta$ for all $x \in V$.

Proof. Put $D=D\left(x_{0}, 2 R\right)$ and $F=D\left(x_{0}, R\right) \backslash V$. Define the stopping times

$$
\begin{gathered}
\tau_{1}=\inf \left\{t>0: B_{t}^{A} \notin D\left(B_{0}^{A}, 2 R\right) \cap V\right\} \\
\sigma_{1}=\inf \left\{t>0: B_{t}^{A} \notin D\left(B_{0}^{A}, 2 R\right)\right\} .
\end{gathered}
$$

From probabilistic potential theory (see [6], Chapter 6, Section 4, or [12])

$$
P_{x_{0}}\left(\tau_{1}<\sigma_{1}\right)=\int_{F} g_{D}\left(x_{0}, y\right) d \mu_{F, D}(y)
$$

Next, let us denote by $\bar{g}_{D}(x, y)$ the Green function of $D$ with respect to the Laplacian $\frac{1}{2} \Delta$. By [20] there exist constants $K_{\lambda}$ and $K_{\lambda}^{\prime}$ depending on the ellipticity constant $\lambda$ such that

$$
K_{\lambda} \bar{g}_{D}(x, y) \leqq g_{D}(x, y) \leqq K_{\lambda}^{\prime} \bar{g}_{D}(x, y) \text { on } F
$$

Thus

$$
P_{x_{0}}\left(\tau_{1}<\sigma_{1}\right) \geqq K_{\lambda} \int_{F} \bar{g}_{D}\left(x_{0}, y\right) d \mu_{F, D}(y) \geqq K_{\lambda, R} \mu_{F, D}(F),
$$

since $\bar{g}_{D}\left(x_{0}, y\right) \geqq$ constant depending on $R$ for all $y \in F$. Thus we conclude that if the capacity condition holds then

$$
P_{x_{0}}\left(\tau_{1}<\sigma_{1}\right) \geqq K_{\lambda, R, \delta}>0
$$

where $K_{\lambda, R, \delta}$ is a constant depending on the parameters indicated.

We now define by induction the stopping times

$$
\tau_{k}=\inf \left\{t>\tau_{k-1}: B_{t}^{A} \notin D\left(B_{\tau_{k-1}}^{A}, 2 R\right) \cap V\right\}
$$

and

$$
\sigma_{k}=\inf \left\{t>\sigma_{k-1}: B_{t}^{A} \notin D\left(B_{\sigma_{k-1}}^{A}, 2 R\right)\right\}
$$

If $\theta_{t}$ denotes the shifting operator, then

$$
\begin{aligned}
\tau_{k-1}+\theta_{\tau_{k-1}} \tau_{1} & =\tau_{k-1}+\inf \left\{r>0: B_{r+\tau_{k-1}}^{A} \notin D\left(B_{\tau_{k-1}}^{A}, 2 R\right) \cap V\right\} \\
& =\inf \left\{r+\tau_{k-1} ; r>0: B_{r+\tau_{k-1}}^{A} \notin D\left(B_{\tau_{k-1}}^{A}, 2 R\right) \cap V\right\} \\
& =\inf \left\{t>0 ; t>\tau_{k-1}: B_{t}^{A} \notin D\left(B_{\tau_{k-1}}^{A}, 2 R\right) \cap V\right\}
\end{aligned}
$$


so that

$$
\tau_{k}=\tau_{k-1}+\theta_{\tau_{k-1}} \tau_{1}
$$

Similarly

$$
\sigma_{k}=\sigma_{k-1}+\theta_{\sigma_{k-1}} \sigma_{1}
$$

Thus the strong Markov property gives

$$
\begin{aligned}
E_{x_{0}}\left(\tau_{k}\right) & =E_{x_{0}}\left(\tau_{k-1}\right)+E_{x_{0}}\left[E_{x_{0}}\left(\theta_{\tau_{k-1}} \tau_{1} \mid \theta_{\tau_{k-1}}\right)\right] \\
& =E_{x_{0}}\left(\tau_{k-1}\right)+E_{x_{0}}\left(E_{B_{\tau_{k}-1}}\left(\tau_{1}\right)\right) \\
& \leqq E_{x_{0}}\left(\tau_{k-1}\right)+\sup _{x \in D} E_{x}\left(\tau_{1}\right) P_{x_{0}}\left(\tau_{k-1}=\sigma_{k-1}\right)
\end{aligned}
$$

It follows from (2.4) and the Itô formula that $E_{x}\left(\sigma_{1}\right) \leqq K_{\lambda}^{\prime} R^{2} / n=K_{\lambda, R, n}^{\prime}$. Therefore we have

$$
E_{x_{0}}\left(\tau_{k}\right) \leqq E_{x_{0}}\left(\tau_{k-1}\right)+K_{\lambda, R, n}^{\prime} P_{x_{0}}\left(\tau_{k-1}=\sigma_{k-1}\right)
$$

Once again, the strong Markov property gives

$$
\begin{aligned}
P_{x_{0}}\left(\tau_{j}=\sigma_{j}\right) & =E_{x_{0}}\left[\chi_{\left(\tau_{j-1}=\sigma_{j-1}\right)} E_{B \tau_{j-1}}\left[\chi_{\left(\tau_{1}=\sigma_{1}\right)}\right]\right] \\
& \leqq\left(1-K_{\lambda, R, \delta}\right) P_{x_{0}}\left(\tau_{j-1}=\sigma_{j-1}\right) \\
& =c_{\lambda, R, \delta} P_{x_{0}}\left(\tau_{j-1}=\sigma_{j-1}\right)
\end{aligned}
$$

with $c_{\lambda, R, \delta}<1$, by (2.5). Combining (2.10) and (2.11) we get

$$
E_{x_{0}}\left(\tau_{k}\right) \leqq E_{x_{0}}\left(\tau_{k-1}\right)+K_{\lambda, R, n}^{\prime}\left[c_{\lambda, R, \delta}\right]^{k}
$$

or

$$
E_{x_{0}}\left(\tau_{k}\right) \leqq K_{\lambda, R, n}^{\prime} \sum_{j=1}^{k}\left[c_{\lambda, R, \delta}\right]^{j}
$$

Since $\tau_{k} \uparrow \tau$ as $k \rightarrow \infty$ a.s. we conclude that

$$
E_{x_{0}}(\tau) \leqq K_{\lambda, R, n}^{\prime} \sum_{j=1}^{\infty}\left[c_{\lambda, R, \delta}\right]^{j}
$$

and the sum converges because $c_{\lambda, R, \delta}<1$. This proves half of the theorem since $x_{0} \in V$ is arbitrary. 
Now suppose there exists a $K<\infty$ such that $E_{x}(\tau)<K$ for all $x \in V$. Fix $x_{0} \in V$. We claim there exist constant $R_{0}$ and $\varepsilon_{0}>0$ depending on $K, \lambda$ and $n$ such that

$$
P_{x}\left\{B_{t}^{A} \text { exits } V \text { before }\left|B_{t}^{A}-x_{0}\right|>\frac{R_{0}}{2}\right\}>\varepsilon_{0} \forall x \in D\left(x_{0}, \frac{R_{0}}{2}\right) \text {. }
$$

To show (2.14) put $D=D\left(x_{0}, R\right)$ and as before $g_{D}$ and $\bar{g}_{D}$ are the Green functions for $D$ with respect to $A$ and $\frac{1}{2} \Delta$, respectively. Then if $\tau_{D}$ is the exit time of $B_{t}^{A}$ from $D$, and if $x \in D\left(x_{0}, R / 2\right)$

$$
\begin{aligned}
E_{x}\left(\tau_{D}\right) & =\int_{D} g_{D}(x, y) d y \geqq K_{\lambda} \int_{D} \bar{g}_{D}(x, y) d y \\
& =K_{\lambda} \frac{R_{0}^{2}-\left|x-x_{0}\right|^{2}}{n} \geqq K_{\lambda} \frac{R_{0}^{2}-\frac{1}{2} R_{0}^{2}}{n}=K_{\lambda} \frac{R_{0}^{2}}{2 n}
\end{aligned}
$$

So if we choose $R_{0}=R_{0}(K)$ large enough,

$$
E_{x}\left(\tau_{D}\right) \geqq 2 K \text { for all } x \in D\left(x_{0}, \frac{R_{0}}{2}\right) .
$$

Now consider $\tau=\tau_{V \cap D}$, the exit time of $V \cap D$. We have

$$
\begin{aligned}
2 K \leqq E_{x}\left(\tau_{D}\right) & =E_{x}\left(\tau+\theta_{\tau} \tau_{D}\right) \\
& =E_{x}(\tau)+E_{x}\left[E_{x}\left(\theta_{\tau} \tau_{D} \mid \mathscr{F}_{\tau}\right)\right] \\
& =E_{x}(\tau)+E_{x}\left[E_{B_{\tau}}\left(\tau_{D}\right)\right] \\
& \leqq K+c E_{x}\left[\frac{R_{0}^{2}-\left|B_{\tau}^{A}-x_{0}\right|^{2}}{n}\right]
\end{aligned}
$$

so that

$$
E_{x}\left[\frac{R_{0}^{2}-\left|B_{t}^{A}-x_{0}\right|^{2}}{n}\right] \geqq K / c
$$

Hence

$$
P_{x}\left[\frac{R_{0}^{2}-\left|B_{\tau}^{A}-x_{0}\right|^{2}}{n} \geqq K / 2 c\right] \geqq \frac{K n}{2 c R_{0}^{2}}
$$

or

$$
P_{x}\left[\left|B_{z}^{A}-x\right|^{2}<R_{0}^{2}-\frac{K n}{2 c R_{0}^{2}}\right] \geqq \frac{K n}{2 c R_{0}^{2}}
$$


from which it follows that

$$
P_{x}\left[\left|B_{\tau}^{A}-x_{0}\right|<R_{0} \cdot c\right] \geqq \varepsilon \text { if }\left|x-x_{0}\right| \leqq R_{0} / 2
$$

where $c<1$ and $\varepsilon>0$ are depending only on $K$, the ellipticity constant, and the dimension. (2.14) now follows from (2.17) and if we set $F=D\left(x_{0}, R_{0} / 2\right) \backslash V$ we find that

$$
\varepsilon<P_{x}\left\{B_{t}^{A} \text { exists } V \text { before }\left|B_{t}^{A}-X_{0}\right|>R_{0} / 2\right\}=\int_{F} g_{D}(x, y) d \mu_{F, D}(y) .
$$

Integrating both sides of the previous inequality over $D\left(x_{0}, R_{0} / 2\right)$ with respect to $x$ and using Fubini's theorem we find that $\mu_{F, D}(F)>\varepsilon$ or

$$
\operatorname{Cap}_{D\left(x_{0}, R_{0}\right)}\left\{D\left(x_{0}, R_{0} / 2\right) \backslash V\right\} \geqq \delta \text { for all } x_{0} \text {. }
$$

where $R_{0}$ depends on $K$, the ellipticity constant $\lambda$, and the dimension $n$. This completes the proof of the Theorem.

Acknowledgements. We are grateful to A. Noell for useful discussions. We also wish to thank the referee for pointing out a sharpening (the present version) of Theorem 1.6. The second named author was partially supported by Norges Almenvitenskapelige Forskningsråd (NAVF), Norway.

Note added in proof. After this paper was finished we became aware of the article by Carl Muller entitled "A characterization of BMO and $\mathrm{BMO}_{p}$ ", published in Studia Math. 72 (1982), 47-57. Muller gives a stochastic proof (for the case of Brownian motion in $\mathbb{C}$ ) of the necessity and sufficiency of the Hayman-Pommerenke/Stegenga condition discussed in Section 2 above.

\section{REFERENCES}

1. M. Aizenman and B. Simon, Brownian motion and a Harnack inequality for Schrödinger operators. Comm, Pure and Appl. Math. 35 (1982), 209-273.

2. H. Alexander, B. A. Taylor and J. L. Ullman, Areas of projections of analytic sets, Invent. Math. 16 (1972), 335-341.

3. D. G. Aronson, Bounds for the fundamental solution of a parabolic equation, Bull. Amer. Math. Soc. 73 (1967), 890-896.

4. S. Axler and J. Shapiro, Putnam's theorem, Alexander's spectral area estimates, and VMO, Math. Ann., to appear.

5. A. Bernard, E. A. Campbell and A. M. Davie, Brownian motion and generalized analytic and inner functions, Ann. Inst. Fourier 29 (1979), 207-228.

6. R. M. Blumenthal and R. K. Getoor, Markov Processes and Potential Theory (Academic Press, 1968).

7. L. Csink and B. ØKsendal, Stochastic harmonic morphisms: Functions mapping the paths of one diffusion into the paths of another, Ann. Inst. Fourier 32 (1983), 219-240.

8. B. Davis, Brownian motion and analytic functions, Ann. Probab. 7 (1979), 913-932. 
9. A Debiard and B. Gaveau, Frontière de Silov de domaines faiblement pseudoconvexes de $\mathbb{C}^{n}$, Bull. Sci. Math. 100 (1976), 17-31.

10. R. Durretr, Brownian Motion and Martingales in Analysis (Wadsworth, 1984).

11. B. Fuglede, Harmonic morphisms between Riemannian manifolds, Ann. Inst. Fourier 28 (1978), 107-144.

12. M Fukushima, Dirichlet Forms and Markov Process (North-Holland/Kodansha, 1980).

13. J. Garnett, Bounded Analytic Functions (Academic Press, 1980).

14. L. J. HAnsen, The Hardy class of a function with slowly-growing area, Proc. Amer. Math. Soc. 45 (1974), 409-410.

15. W. K. Hayman and Ch. Pommerenke, On analytic functions of bounded mean oscillation, Bull. London Math. Soc. 10 (1978), 219-224.

16. N. Ikeda and S. Watanabe, Stochastic Differential Equations and Diffusion Process (NorthHolland/Kodansha, 1981).

17. S. Kakutani, Two dimensional Brownian motion and the type problem of Riemann surfaces, Proc. Japan Acad. 21 (1945), 138-140.

18. S. KRANTZ, Holomorphic functions of bounded mean oscillation and mapping properties of the Szego projection, Duke Math. J. 49 (1980), 743-761.

19. S. Kobayashi, Range sets and BMO norms of analytic functions, Canad. J. Math. 36 (1984), 747-755.

20. W. Littman, G. Stampacchia and H. F. Weinberger, Regular points for elliptic equations with discontinuous coefficients, Ann. Scuola. Norm. Sup. Pisa. CP. Sci. 17 (1963), 43-76.

21. E. Low, Inner functions and boundary values in $H^{\infty}(\Omega)$ and $A(\Omega)$ in smoothly bounded pseudoconvex domains, Math. Z. 185 (1984), 191-210.

22. H. P. McKean, Stochastic Integrals (Academic Press, 1969).

23. B. ØKSENDAL, When is a stochastic integral a time change of a diffusion? to appear.

24. D. A. Stegenga, A geometric condition which implies BMOA, Michigan Math. J. 29 (1980), 247-252.

25. E. M. Stein, Boundary Behaviour of Holomorphic Functions of Several Complex Variables (Princeton Univ. Press, 1972).

Department of Mathematics 253-37

California Institute of Technology

Pasadena, CA 91125
Department of Mathematics

University of California, Los Angeles Los ANgeles, CA 90024

Current address

Department of Mathematics

UNIVERSITY OF OSLO

BOX 1053 BLINDERN

N-0316 OsLo 3

NoRWAY 